\title{
Rates of hospitalization and death for all-cause and rotavirus acute gastroenteritis before rotavirus vaccine introduction in Kenya, 2010-2013
}

Richard Omore ${ }^{1,2^{*}}$ (D), Sammy Khagayi ${ }^{1}$, Billy Ogwel ${ }^{1}$, Reuben Onkoba ${ }^{1}$, John B. Ochieng ${ }^{1}$, Jane Juma ${ }^{1}$, Stephen Munga ${ }^{1}$, Collins Tabu ${ }^{3}$, Sergon Kibet ${ }^{4}$, J. Pekka Nuorti², Frank Odhiambo ${ }^{1}$, Jason M. Mwenda ${ }^{5}$, Robert F. Breiman ${ }^{7}$, Umesh D. Parashar ${ }^{6}$ and Jacqueline E. Tate ${ }^{6}$

\begin{abstract}
Background: Rotavirus vaccine was introduced in Kenya immunization program in July 2014. Pre-vaccine disease burden estimates are important for assessing vaccine impact.

Methods: Children with acute gastroenteritis (AGE) ( $\geq 3$ loose stools and/or $\geq 1$ episode of unexplained vomiting followed by loose stool within a 24-h period), hospitalized in Siaya County Referral Hospital (SCRH) from January 2010 through December 2013 were enrolled. Stool specimens were tested for rotavirus (RV) using an enzyme immunoassay (EIA). Hospitalization rates were calculated using person-years of observation (PYO) from the Health Demographic Surveillance System (HDSS) as a denominator, while adjusting for healthcare utilization at household level and proportion of stool specimen collected from patients who met the case definition at the surveillance hospital. Mortality rates were calculated using PYO as the denominator and number of deaths estimated using total deaths in the HDSS, proportion of deaths attributed to diarrhoea by verbal autopsy (VA) and percent positive for rotavirus AGE (RVAGE) hospitalizations.

Results: Of 7760 all-cause hospitalizations among children < 5 years of age, 3793 (49\%) were included in the analysis. Of these, 21\% (805) had AGE; RV was detected in 143 (26\%) of 541 stools tested. Among children < 5 years, the estimated hospitalization rates per 100,000 PYO for AGE and RVAGE were 2413 and 429, respectively. Mortality rate associated with AGE and RVAGE were 176 and 45 per 100,000 PYO, respectively.
\end{abstract}

Conclusion: AGE and RVAGE caused substantial health care burden (hospitalizations and deaths) before rotavirus vaccine introduction in Kenya.

Keywords: Rotavirus, Morbidity, Mortality, Children, Kenyan

\section{Background}

Rotavirus is the most common cause of vaccine-preventable severe acute gastroenteritis (AGE) among infants and young children worldwide [1, 2]. In 2013, RVAGE was estimated to cause 215,000 global deaths among children $<5$ years of

\footnotetext{
* Correspondence: Omorerichard@gmail.com

${ }^{1}$ Kenya Medical Research Institute, Center for Global Health Research (KEMRI-CGHR), Kisumu, Kenya

${ }^{2}$ Health Sciences Unit, Faculty of Social Sciences, University of Tampere,

Tampere, Finland

Full list of author information is available at the end of the article
}

whom $2 \%(\sim 4000)$ were from Kenya [3] alone. In Kenya, RVAGE accounts for 19\% ( 9000) of annual hospitalizations among children < 5 years [4]. Two RV vaccines Rotarix ${ }^{\circ}$ (GlaxoSmithKline), and RotaTeq ${ }^{\circ}$ (Merck \& Co.), are approved and recommended by the World Health Organization (WHO) for global use [5]. Efficacy and effectiveness studies of these vaccines have shown significant reduction in AGE and RVAGE associated hospitalizations and deaths among children $<5$ years in both clinical trials and in settings where they have been incorporated into the national immunization programs [1, 6-10]. Consistent with data

(c) The Author(s). 2019 Open Access This article is distributed under the terms of the Creative Commons Attribution 4.0 International License (http://creativecommons.org/licenses/by/4.0/), which permits unrestricted use, distribution, and 
from Mexico [6] and Brazil [7, 11], African countries that were early introducers of RV vaccines including Malawi [10], Ghana [12], and Rwanda [13], have shown remarkable declines in childhood morbidity and mortality associated with AGE and RVAGE. Furthermore, the cost benefit of these vaccines has equally been demonstrated $[4,7,14]$ .RV vaccine (Rotarix ${ }^{\oplus}$ ) was introduced into the Kenya national immunization program in July 2014. Recent population-based data on pre-vaccine disease rates are not available in Kenya. However, such data are needed to evaluate the impact of vaccination program and may help county and national level governments, regional and global decision makers with evidence needed to support investment in these vaccines.

We examined baseline rates of AGE and RVAGE specific hospitalizations and deaths among children $<5$ years in rural western Kenya from January 1, 2010 to December 31, 2013 before RV vaccine introduction in Kenya.

\section{Methods \\ Study site}

Rotavirus surveillance and the Health Demographic Surveillance System (HDSS) platform in our study setting has been detailed elsewhere $[15,16]$. In brief, the HDSS site is located in Siaya County in rural western Kenya. The HDSS is a longitudinal study that monitors births, deaths, out and in-migrations and other demographics of a defined population [16]. Our study was conducted in Karemo HDSS area within Siaya county referral hospital $(\mathrm{SCRH})$ - the main regional referral hospital in this setting.

\section{Rotavirus surveillance and laboratory methods}

As part of the African-based, World Health Organization (WHO) coordinated RV rotavirus disease surveillance network [17], we conducted hospital-based prospective surveillance for RVAGE within the Kenya Medical Research Institute (KEMRI) operated HDSS area in Karemo [15]. Children aged 0-59 months residents of Karemo HDSS, hospitalized at the in-patient department of SCRH with AGE; defined as $\geq 3$ looser than normal stools and/or $\geq 1$ episode of unexplained vomiting followed by loose stool within a 24-h period beginning within the 7 days before seeking healthcare from January 1, 2010 to December 31, 2013 were eligible for enrolment. Trained health facility recorders approached all eligible patient children, explained the study and administered a questionnaire on demographics to their caretakers after obtaining informed consent. A study clinician then examined these patients and administered the standardized questionnaire to their parent/caretaker to gather information about symptoms, medical history, laboratory investigations, diagnosis, treatment and outcome of hospitalization. A whole stool specimen was collected from each participant in a plastic diaper from which at least $2 \mathrm{ml}$ of stool was scooped into a specimen container using a sterile spatula within $48 \mathrm{~h}$ of admission, transported on the same day to the enteric laboratory based at the KEMRI-CGHR, and finally tested for rotavirus using a commercial enzyme immunoassay (EIA) (Rotaclone Kit, Meridian Bioscience). A case of RVAGE was defined as an AGE patient with a RV positive stool specimen.

\section{Data management}

Details of the enrolment, testing and data management have been described previously [15]. In brief, we linked clinic data to laboratory results and to the longitudinal data including cause of death from verbal autopsy (VA) from the HDSS. During data collection, built-in software in the electronic questionnaire with built-in checks and controls ensured quality control. The linked data were then uploaded and managed using a Microsoft SQL Server 2008 database. Data were analysed using SAS version 9.4 (SAS Institute, Inc. Cary, North Carolina, USA).

\section{Statistical analysis Descriptive analysis for AGE and RVAGE}

Proportion of admissions due to AGE was calculated by dividing the number of AGE cases by the number of all-cause admissions at SCRH who were residents of Karemo HDSS during the study period. The proportion of admissions that were associated with RVAGE was calculated by dividing the number of RV positive stool samples with the total samples collected and tested. Positivity rates by month and patient characteristics (age, gender, clinical features and illness outcome) were calculated. These proportions were plotted by month to show seasonality.

\section{Analysis of RVAGE, disease severity and risk factors}

The severity of RVAGE was assessed by using the 20-point Vesikari score [18].A score of less than 11 was categorized as mild while a score of 11 or more was classified as severe. Bivariate comparison of the laboratory-confirmed RV positivity and patient characteristics and treatment outcomes were evaluated using chi-square tests.

\section{Incidence rates of hospitalization and mortality due to AGE and RVAGE}

We used person-years of observation (PYO) contributed by all children aged less than 5 years residents of Karemo region during the study period as the denominator. As described previously [15], we calculated PYO by totaling person-time for all children aged 0-59 months who met HDSS residency requirement during the 4-year study period from 1st January 2010 or date of enrolment (if after) until they exited or lost their HDSS residency status through out-migration or death. 
The crude hospitalization rates were calculated by dividing the total number of AGE and RVAGE hospitalizations by the PYO contributed by children aged 0-59 months for the period that they met residency criteria for the HDSS.

We used two adjustments for the hospitalization rates. First, to account for possible missed AGE cases, we divided the crude rate of AGE and RVAGE by the proportion of all in-patients who met the stool collection criteria, whether a sample was collected or not.

The second adjustment accounted for children with AGE or possibly RVAGE who did not reach or attend a sentinel health care facility as reported from a population-based, healthcare utilization and attitude surveys (HUAS) for diarrhoea-a separate household survey conducted within the HDSS during the current RVAGE surveillance period [19].The HUAS revealed that the frequencies of seeking care for moderate-to-severe diarrhoea (MSD) from a hospital were $69,70,67,57$ and $64 \%$ for children aged 0-5, 611, 12-23, 24-59 and 0-59 months, respectively (GEMS-Kenya unpublished data). The $95 \%$ confidence intervals (CI) were calculated around crude rates by using the PEPI method [20]. Crude rates were then adjusted using Delta method [21, 22]. The adjusted hospitalization rates were finally stratified and reported by age groups that included; $0-5,6-11,12-23,24-59,0-11$ and $0-59$ months.

\section{AGE and RVAGE mortality rates}

Deaths were recorded at household level through regular interviews of HDSS residents. Diarrhoea as a cause of death was derived from Verbal autopsy (VA). The VA methodologies, coding and interpretation are described elsewhere [23, 24]. Upon the death of an HDSS resident, a trained village-based reporter sent a notification to HDSS data team. After a mourning period of at least 3 weeks, the interviewer from the HDSS approached the most appropriate interviewee who was closest to the deceased to administer a detailed questionnaire focusing on the signs, symptoms and medical history of the deceased. The VA data were collected electronically, validated and processed using an InterVA program, which is a probabilistic computer-based expert opinion algorithm that determines the most probable cause of death as described elsewhere [24]. We calculated the number of deaths attributed to RV by multiplying the total under-five deaths among HDSS residents in the study area by the proportion of deaths attributable to diarrhoea by VA, and the proportion of hospitalized AGE episodes attributable to RV in each of the various age groups as described below.
Mortality rates associated with rotavirus gastroenteritis were obtained by dividing the number of deaths attributed to rotavirus by the total PYO in each of the specific age groups as described above.

\section{Results}

\section{Enrolment profile and patient characteristics}

During the study period, a total of 7760 all-cause hospitalizations among children $<5$ years of age were recorded at the SCRH paediatric ward, out of which 3793 were Karemo HDSS resident population. Among the 3793 Karemo HDSS resident children, 805 (21, 95\% CI: 20-23) children were hospitalized due to AGE (Fig. 1). RV-positivity among hospitalized children from Karemo with AGE was more pronounced in infants ( $<12$ months of age), then toddlers (12-23 months of age), and was least in school-age children (24-59 months of age) (Table 1). Characteristics of patients who had stool specimens collected and those who did not have specimens collected are shown in Table 2.

Of the 541 stool samples collected, 204 (38\%) were from infants aged 6-11 months. There was no difference in stool collection by gender. Furthermore, we did not observe any statistical difference in rotavirus positivity in male versus female patients among infants aged $<12$ months $((69 / 211[32.7 \%])$ vs. $(42 / 165[25.4 \%]), \mathrm{OR}=$ 1.42, $p=0.13)$,toddlers aged $12-23$ months $((9 / 61$ [14.7\%]) vs. $(12 / 46[26.1 \%]), \mathrm{OR}=0.49, p=0.15)$, or in children aged $24-59$ months $((9 / 37$ [24.3\%]) vs. $(2 / 21$ [9.5\%]), OR $=3.05, p=0.18)$, respectively.

The overall annual proportion of rotavirus detection ranged from $43 / 147(29.3 \%)$ in 2010 to $21 / 95$ (22.1\%) in 2013 and the annual proportion of samples detected with rotavirus did not differ significantly over the 4-year study period. Rotavirus hospitalizations were seen throughout the year over the surveillance period, but peaked from January through March and around August-September each year during study period (Fig. 2).

Compared with non-RVAGE cases, RVAGE cases were younger ((median age $=8$ Interquartile range [IQR] 512) vs. 9 [IQR: 6-15] months; $p<0.032)$ ), more likely to present with vomiting ((126/143 (88.1\%) vs. 297/397 (74.8\%)), and more likely to be classified as severe by Vesikari score $(88 / 143(61.5 \%)$ vs. $179 / 398$ (44.9\%), $p$-value $=0.0007)$. (Table 3$)$.The length of hospitalization was similar for RVAGE compared to non-RVAGE (number of hospitalization days 4 [IQR] 3-6 vs. 4 [IQR] 3-6, $p$-value $=0.564)$. 


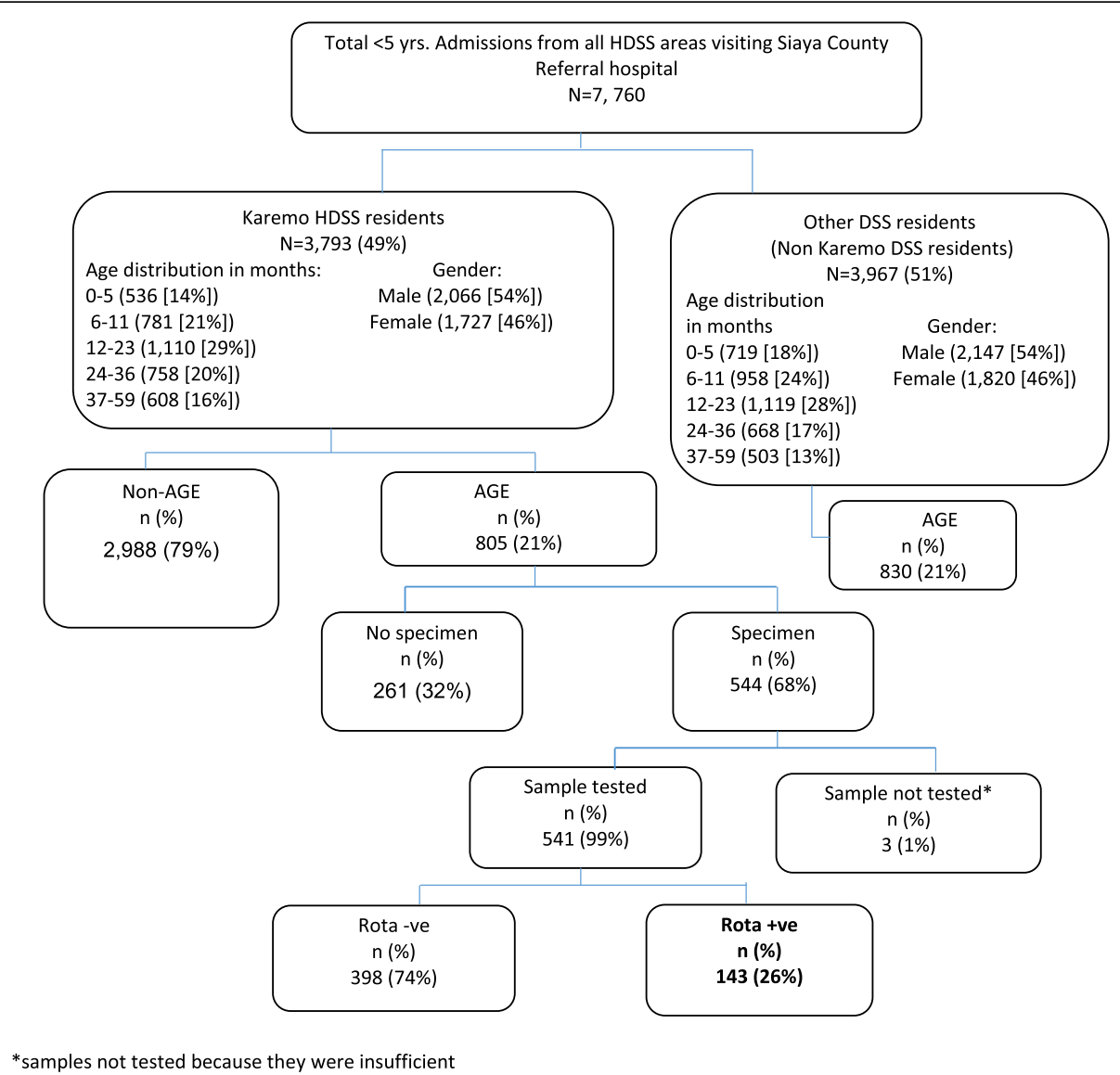

Fig. 1 Flow diagram of Karemo DSS residents $<5$ yrs. who were hospitalized and enrolled in the study from Siaya county referral hospital, western Kenya 2010-2013

Table 1 Characteristics of children< 5 years hospitalized at SCRH with all cause morbidity, AGE and RVAGE, 2010-2013

\begin{tabular}{|c|c|c|c|c|}
\hline \multirow[t]{2}{*}{ Characteristics } & $\begin{array}{l}\text { All Cause Hospitalizations } \\
\text { from Karemo DSS ( } N=3793)\end{array}$ & $\begin{array}{l}\text { AGE Hospitalizations } \\
(N=805)\end{array}$ & $\begin{array}{l}\text { AGE specimen collected and tested } \\
\text { for } R V \not(N=541)\end{array}$ & $\begin{array}{l}\text { Proportion tested RV Positive } \\
(N=143)\end{array}$ \\
\hline & $n$ & n (row \%) & n (row \%) & n (row \%) \\
\hline \multicolumn{5}{|l|}{ Sex } \\
\hline Male & 2066 & 461 (22\%) & 309 (67\%) & 87 (28\%) \\
\hline Female & 3793 & $344(20 \%)$ & 232 (67\%) & $56(24 \%)$ \\
\hline \multicolumn{5}{|l|}{ Year } \\
\hline 2010 & 920 & $224(24 \%)$ & 147 (66\%) & $43(29 \%)$ \\
\hline 2011 & 1335 & $313(23 \%)$ & $206(66 \%)$ & $53(26 \%)$ \\
\hline 2012 & 822 & 147 (18\%) & 93 (63\%) & $26(28 \%)$ \\
\hline 2013 & 716 & $121(17 \%)$ & 95 (79\%) & $21(22 \%)$ \\
\hline \multicolumn{5}{|l|}{ Age (Months) } \\
\hline $0-5$ & 536 & $205(38 \%)$ & $141(69 \%)$ & $45(32 \%)$ \\
\hline $6-11$ & 781 & $285(36 \%)$ & $204(72 \%)$ & 57 (28\%) \\
\hline $12-23$ & 1110 & $205(18 \%)$ & 138 (67\%) & 30 (22\%) \\
\hline $24-36$ & 687 & $70(10 \%)$ & 40 (57\%) & $9(23 \%)$ \\
\hline $37-59$ & 679 & $40(6 \%)$ & $18(45 \%)$ & $2(11 \%)$ \\
\hline
\end{tabular}


Table 2 Characteristics of Karemo resident children $<5$ years hospitalized with AGE who had stool collected and those without stool collected, Siaya County Referral Hospital, Western Kenya, 2010-2013

\begin{tabular}{lll}
\hline Characteristic & $\begin{array}{l}\text { Stool Sample Collected } \\
(N=544) \\
\mathrm{n}(\%)\end{array}$ & $\begin{array}{l}\text { Stool Sample Not collected } \\
(N=261) \\
\mathrm{n}(\%)\end{array}$ \\
\hline Age & $143(26)$ & $62(24)$ \\
$0-5$ months & $204(38)$ & $81(31)$ \\
$6-11$ months & $139(26)$ & $66(25)$ \\
$12-23$ months & $58(11)$ & $52(20)$ \\
$24-59$ months & & $149(57)$ \\
Gender & $312(57)$ & $112(43)$ \\
Male & $232(43)$ & \\
Female & & $93(36)$ \\
Vesikari Score & $268(49)$ & $168(64)$ \\
Severe & $276(51)$ & \\
Mild & & 0.9034 \\
\hline
\end{tabular}

\section{Hospitalization attributed to AGE in Karemo HDSS}

The highest annual hospitalization rate (per 100,000 PYO) associated with AGE was observed in 2011 followed by 2010, 2012 and 2013 in descending order. The annual incidence (per 100,000) of hospitalizations due to all cause AGE was highest among infants and children aged 6-11 months remained most affected.

\section{Hospitalization attributed to RVAGE among children $<5$ years from Karemo HDSS}

Incidence rates of RVAGE associated hospitalization was highest among infants, particularly among those aged 6-11 months. We observed the highest RVAGE hospitalization rate in 2011 followed by 2010, 2012 and 2013 in decreasing order. Hospitalization rates for AGE and RVAGE are shown in Table 4.

\section{Mortality attributed to AGE and RVAGE}

Discharge information was available for 531 (98\%) of the hospitalizations due to AGE of whom 33 (6.2\%) died during hospitalization. The case-fatality proportion among RVAGE ((4.2\%), [6/142]) compared to that observed from non-RVAGE ((6.9\%), [27/389]) cases was similar, $p=0.26$.

The highest mortality rates of AGE and RVAGE were observed among infants (<12 months of age), and remained most elevated among infants aged 6-11 months. Annual mortality rates associated with RVAGE were stable between 2010 and 2011, but increased before

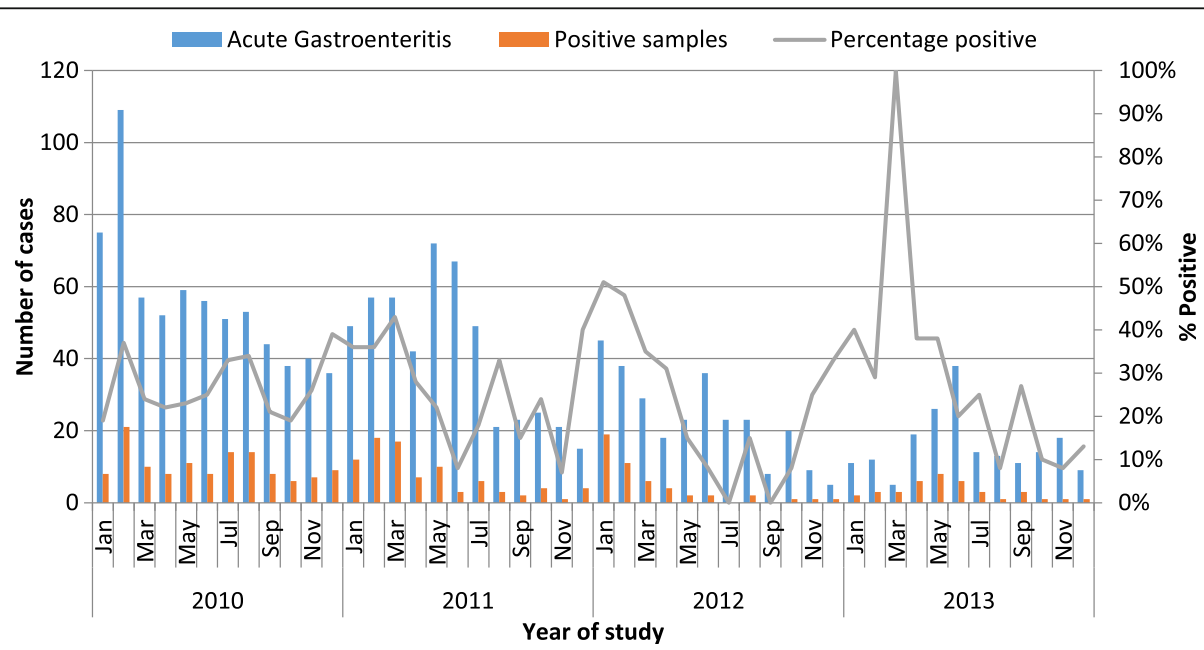

Fig. 2 Rotavirus infection trends among hospitalized children $<5$ years from Karemo DSS resident population seeking Care from Siaya County Referral Hospital, western Kenya 2010-2013 
Table 3 The Vesikari scores for severity of illness among RVAGE and non- RVAGE hospitalized children $<5$ yrs. in Siaya County Referral Hospital, western Kenya, 2010-2013

\begin{tabular}{|c|c|c|c|c|}
\hline \multirow[t]{2}{*}{ Variable } & \multirow[t]{2}{*}{ Points assigned } & \multirow{2}{*}{$\begin{array}{l}\text { Rotavirus-positive } \\
N=143\end{array}$} & \multirow{2}{*}{$\begin{array}{l}\text { Rotavirus-negative } \\
N=398\end{array}$} & \multirow[t]{2}{*}{$P$-value } \\
\hline & & & & \\
\hline \multicolumn{5}{|l|}{ Duration of diarrhea (days) } \\
\hline $1-4$ & 1 & $119(83)$ & $281(70 \%)$ & \multirow[t]{3}{*}{0.0089} \\
\hline 5 & 2 & $4(3 \%)$ & $31(8 \%)$ & \\
\hline$>=6$ & 3 & $20(14 \%)$ & $86(22 \%)$ & \\
\hline \multicolumn{5}{|l|}{ Max no. diarrhea/24 h } \\
\hline $1-3$ & 1 & $41(29 \%)$ & $153(38 \%)$ & \multirow[t]{3}{*}{0.1091} \\
\hline $4-5$ & 2 & $75(52 \%)$ & 177 (45\%) & \\
\hline$>=6$ & 3 & $27(19)$ & $68(17 \%)$ & \\
\hline Duration of vomiting (days) & & $N=126$ & $N=296$ & \\
\hline 1 & 1 & $22(15.4)$ & $77(19.4)$ & \multirow[t]{3}{*}{0.165} \\
\hline 2 & 2 & $27(18.9)$ & $57(14.4)$ & \\
\hline$>=3$ & 3 & 77 (53.8) & $162(40.9)$ & \\
\hline \multicolumn{5}{|l|}{ Max no. vomiting/24 h } \\
\hline 1 & 1 & $8(7 \%)$ & $39(13 \%)$ & \multirow[t]{3}{*}{0.1003} \\
\hline $2-4$ & 2 & $95(75 \%)$ & $214(72 \%)$ & \\
\hline$>=5$ & 3 & $23(18 \%)$ & $43(15 \%)$ & \\
\hline \multicolumn{5}{|l|}{ Fever } \\
\hline$<37.0$ & 0 & $74(52 \%)$ & $196(49 \%)$ & \multirow[t]{4}{*}{0.0435} \\
\hline $37.1-38.4$ & 1 & $58(40 \%)$ & 157 (40\%) & \\
\hline $38.5-38.9$ & 2 & $8(6 \%)$ & 12 (3\%) & \\
\hline$>=39$ & 3 & $3(2 \%)$ & $33(8 \%)$ & \\
\hline Dehydration & & $N=55$ & $N=139$ & \\
\hline $1-5 \%$ & 2 & 48 (87\%) & 117 (84\%) & \multirow[t]{2}{*}{0.5852} \\
\hline$>=6 \%$ & 3 & 7 (13\%) & $22(16 \%)$ & \\
\hline Treatment* & 2 & $143(100 \%)$ & 398 (100\%) & - \\
\hline
\end{tabular}

${ }^{*}$ All cases were treated in the ward

RV vaccine introduction, especially among children aged 6-11 months. Mortality rates attributed to AGE and RVAGE are shown in Table 5.

\section{Discussion}

This study documents comprehensive, age-stratified population-based hospitalization and mortality rates associated with AGE and RVAGE before introduction of $\mathrm{RV}$ vaccines among Kenyan children $<5$ years in a rural community whose demographic and healthcare seeking characteristics are well described [16, 19]. Unlike other WHO rotavirus surveillance study sites in Africa, our hospital-based surveillance site for rotavirus is unique for a few reasons. First, it is supported by an ongoing HDSS which monitors population denominators and conducts verbal autopsy [16]. Second, our surveillance hospital is the only regional public referral hospital for the local HDSS and the only in-patient facility within the HDSS, making our surveillance data representative of the population as shown from our current data and as previously observed [15]. Furthermore, the advantages of a population-based incidence rate are two-fold. First, they provide an opportunity to estimate number of people affected by a disease. Second, they can help to project the number of illness episodes that can be prevented with effectively known interventions such as vaccines [25].

Our 4-year study's most important findings are that before RV vaccine introduction in Kenya; approximately 90 and $60 \%$ of RVAGE hospitalized children were aged $<2$ years and $<1$ year, respectively, and that hospitalizations and mortality associated with AGE and RVAGE were highest among infants. Furthermore our data suggests that children bearing the greatest burden of morbidity and mortality associated with AGE and RVAGE were infants aged 6-11 months. This finding is similar to observations from neighboring Sudan where pre-RV vaccine data indicates that 91 and $61 \%$ of rotavirus hospitalizations occurred before 2 years and 1 year respectively [26]. 


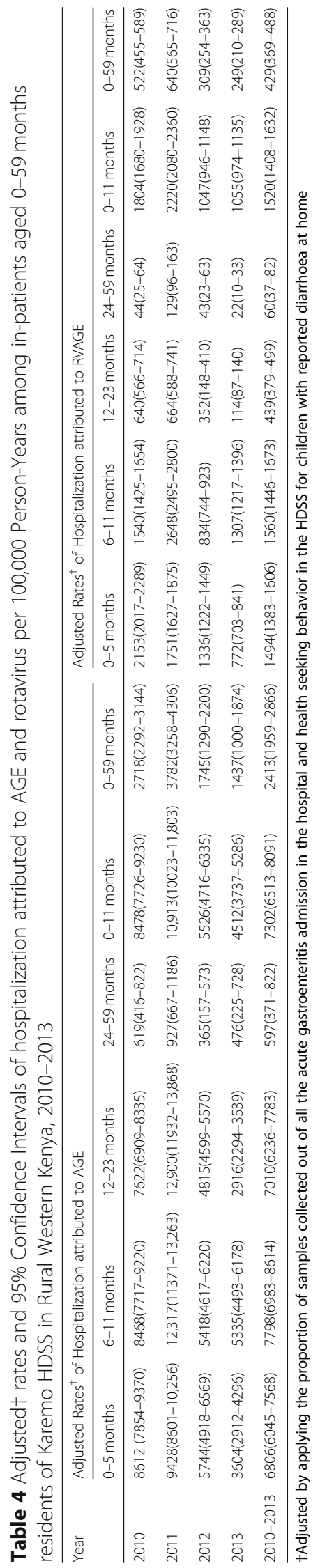




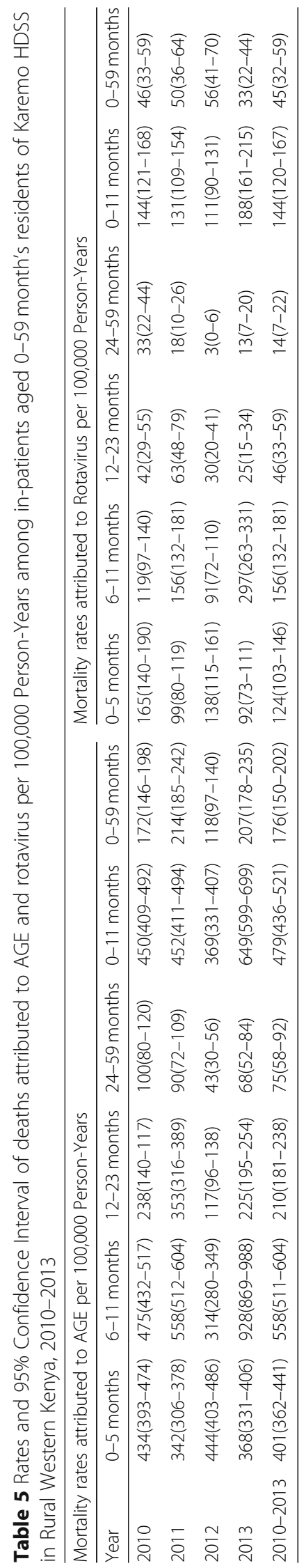


Furthermore, our finding is consistent with observations from the first 2 years of the current study [15], a study conducted at the coastal region of Kenya [27], Global Enteric Multicenter Study (GEMS) [2] and other studies conducted in Europe [28] before introduction of RV vaccines. Our observation that $21 \%$ of hospitalizations among children $<5$ years in the HDSS were due to AGE is similar to $23 \%$ reported previously from mid-term analysis of our current study [15], 22\% reported from Kilifi HDSS in coastal region of Kenya [27], 21\% from neighboring Mwanza region in Tanzania [29], and 21\% from Ethiopia [30]. In addition, our finding that $26 \%$ of hospitalized AGE case patients were infected with RV remains similar to the rate of $27 \%$ reported from mid-term analysis of our current surveillance data [15] and to 29\% from Kilifi HDSS at the Kenyan coast [27]. These observations suggest that AGE and RVAGE burden in our setting is comparable to those from other settings in Kenya and neighboring countries before RV vaccine introduction. Our observation that rates of hospitalization due AGE and RVAGE declined over the study period before vaccine introduction may be associated with unknown non-RV vaccine intervention factors. However, the proportion of all deaths that were associated with AGE and RVAGE did not follow the same pattern. Thus, these observed trends are difficult to explain, though in part may reflect the effects of other interventions. Although widespread distribution and use of zinc and ORS as part of devolved government development efforts in Kenya has been described [31] and may be a contributing factor to the observed decline in diarrhea burden in this setting, such argument remains speculative and prompts further investigation. This trend however is consistent with other observations from a recent community-based survey conducted in this setting [19], and is not dissimilar to the global trend of diarrhoea and rotavirus disease [3, 32].

Our data show that rotavirus was more commonly detected among infants. Moreover, RVAGE presented with more severe episodes than non-RVAGE as characterized by severe dehydration, vomiting and low grade fever -an observation similar to other previous studies [30, 33-35]. Rotavirus is the most common cause of severe dehydrating diarrhoea and is the leading pathogen associated with moderate-to-severe diarrhoea (MSD) [35], as further reaffirmed by GEMS - the largest diarrhoea etiology case-control study ever conducted in countries representing the highest disease burden regions located in Africa and Asia [2]. Severe dehydration caused by diarrhoea in children is a major cause of preventable morbidity and mortality in Kenya [31]. As commonly observed in our setting and consistent with the caretakers healthcare seeking trends in Kenya [19, 31], delay in seeking care for childhood diarrhoea and reducing amount of fluid and food intake during childhood AGE illness can lead to severe disease. Our current study found that case-fatality among RVAGE was not significantly different from non-RVAGE cases, suggesting that rotavirus may not be associated with mortality in hospital based studies as shown from other studies [33, 36]. This finding supports the assumption that seeking care for RVAGE from a health care facility enables access to appropriate rehydration, which would then reduce the risk of death from the disease.

Understanding seasonality of rotavirus can help formulate hypothesis for assessing potential factors influencing transmission and guide policy makers in deciding on appropriate interventions and approaches that can work in local settings for improving case management during peak seasons [37]. For example, in settings such as USA, rotavirus seasons have been observed to be delayed, shortened, and diminished [4, 38] after vaccine introduction. In our current analysis, rotavirus detection peaked in months which are locally known to be usually warm and dry. Our current findings are consistent with recent observations from Kenya [15, 33], and remains similar to findings from other studies conducted in Burkina Faso [37], Peru and Bangladesh [39] before rotavirus vaccine introduction in those settings. Although there is no unifying rotavirus seasonality pattern globally [40], it's spread by the faecal-oral route remains agreeable [35], and even airborne or droplet transmission has been postulated [41]. The later attribute potentially makes the virus transmission route also to resemble that of other non-enteric respiratory infectious diseases such as measles [42].These observations suggests that a drop in humidity and rainfall combined with dry soil could potentially increase additional chance for transmission through aerial contaminated faecal materials since survival of rotavirus may still be favored in such conditions as described elsewhere [41, 43].

Treating RVAGE is expensive. In Kenya, it has been estimated that rotavirus disease cost the national healthcare system \$10.8 million each year, and that a 2-dose rotavirus vaccine (RVV) series can avert $\sim 2500$ deaths, $\sim 6000$ hospitalizations and $\sim 860,000$ clinic visits with a cost saving of $\$ 2.1$ million annually [4].RV vaccines have been shown to be effective in reducing the hospitalizations and death due to diarrhoea in children and the protective effect potentially lasts through 2 nd year of life $[1,44]$.While the benefit of these vaccines has been documented in other African countries where they were introduced ahead of Kenya, such as in South Africa [45], Rwanda [3], Ghana [12], and Togo [46], population-level benefits of RVV are yet to be demonstrated from Kenya.

A possible limitation of our current study is that many rotavirus-associated fatalities are likely associated with delay in healthcare seeking [5]. Furthermore, VA relies on signs, symptoms and circumstances prior to death to 
assign cause of death which is subject to misclassification error, and therefore the method as applied in our current study may lead to over or under-estimation of mortality [36]. Our methodology for estimating diarrhoea deaths attributable to rotavirus was based on the following 3 assumptions: (i) that in the absence of treatment, the hospitalized severe cases would not have survived; (ii) the treatment effect on survival of severe diarrhoea is equal for rotavirus and non-rotavirus diarrhoea; and that (iii) the rotavirus attributable fraction of severe diarrhoea observed in the sentinel hospital are generalizable to the source population within each age stratum as already described elsewhere [36]. Maintaining caution when interpreting these estimates is important since we recognize that such assumptions may affect the validity and generalizability of the estimates to the general population. However, since there are currently no reliable data for the direct measurement of the proportion of diarrhoea deaths that are attributable to rotavirus $[22,36]$ especially in the high disease burden regions located mostly in low-and middle income countries such as in our setting [3], we believe our methodology remains more reasonable, robust and applicable as recommended by WHO [22, 36]. Moreover, our current hospital surveillance data suggests agreeable representation of the source population consistent with previous observations [15].

\section{Conclusions}

This study shows that AGE and RVAGE associated hospitalization and deaths are high in this setting with children aged 6-11 months bearing the greatest burden. These findings support the introduction of a vaccine that would potentially provide protection to young children before the disease peaks at $6-11$ months of age in this setting. While improvements in drinking water, sanitation and hygiene can effectively prevent other forms of diarrhoea, such interventions do not adequately prevent the spread of rotavirus, thus leaving vaccines as the best alternative in preventing AGE and RVAGE in settings such as ours [5]. Continued surveillance will be important for measuring the impact of rotavirus vaccine introduction in Kenya.

\section{Abbreviations}

AGE: Acute gastroenteritis; CDC: US Centers for Disease Control and Prevention; CGHR: Center for Global Health Research; EIA: Enzyme immunoassay; GEMS: Global Enteric Multi-Center Study; HDSS: Health Demographic Surveillance System; KEMRI: Kenya Medical Research Institute; $\mathrm{MoH}$ : Ministry of Health; PYO: Person-years of observation; RVAGE: Rotavirus AGE; SAS: Statistical Analysis Software; SCRH: Siaya county referral hospital; VA: Verbal autopsy; WHO: World Health Organization

\section{Acknowledgements}

This study includes data generated by the KEMRI and CDC joint operated HDSS, which is a member of the International Network for the Demographic Evaluation of Populations and their Health (INDEPTH). We acknowledge the contributions of and thanks to; WHO country office in Kenya and WHO-AFRO office based in Brazzaville; thanks to Ministry of Health staff for supporting the data collection and processing; the entire KEMRI and CDC study team at SCRH. Special thanks to Dr. Daniel R. Feikin and, Dr. Kayla F. Laserson (CDC), Dr. Amek Nyagwara (KEMRI-CGHR), Charles Mwitherero (WHO), Caroline Maina (MoH, Kenya), Alice Ngereso (WHO), Prof. Kirsi Lumbe Sandat, Tiina Kangasala and Catarina. Stahle-Nieminen (University of Tampere), Linet Aluoch Sewe, Collins Okello, Pamela Kanga, Peter Jaron and Ken Ruttoh (KEMRI-CGHR) for supporting the study operations. We are grateful to the caretakers in the Karemo, Asembo and Gem communities who participated in this work. This manuscript is published with the approval of the Director, KEMRI.

\section{Funding}

The study was funded and supported by grants from the GAVI alliance through WHO, the CDC Division of Viral Diseases, US Centers for Disease Control and Prevention, Atlanta, GA, USA Branch program funds through the Kenya Medical Research Institute. Richard Omore was supported in part by the International Doctoral Programme in Epidemiology and Public Health (IPPE), University of Tampere, Finland. The funders did not play any role in the study and interpretation of its outcome.

\section{Availability of data and materials}

Data were obtained with permission of the KEMRI and CDC HDSS Steering committee. Any data requests may be sent to the above steering committee, through the corresponding author.

\section{Disclaimer}

The findings and conclusions in this report are the findings and conclusions of the authors and do not necessarily represent the official position of the Kenya Medical Research Institute or the US Centers for Disease Control and Prevention.

\section{Authors' contributions}

Conceived and designed the study: RO1, SK1, RO2, JPN, RFB, JMW, UDP, JT. Performed the study: RO1, SK1, BO, RO2, JBO, JJ, SM, CT, SK2, FO, RFB, UDP, JT. Analyzed the data: RO1, SK1, BO, RO2, JBO, JT. Contributed reagents, materials/analysis tools: ALL. Wrote the paper: RO1, SK1, JT. Reviewed the manuscript: All authors. Interpretation of data and critical revision of the manuscript for important intellectual content: RO1, SK1, JPN, RFB, UDP, JT. All authors read and approved the final manuscript.

Ethics approval and consent to participate

Written informed consent was obtained from all the guardians or caretakers of the children before enrolment into the study. This study was approved as part of the HDSS by both the Ethical Review Committee of the Kenya Medical Research Institute and CDC-Atlanta.

Consent for publication

Not applicable.

Competing interests

None declared.

\section{Publisher's Note}

Springer Nature remains neutral with regard to jurisdictional claims in published maps and institutional affiliations.

\section{Author details}

${ }^{1}$ Kenya Medical Research Institute, Center for Global Health Research (KEMRI-CGHR), Kisumu, Kenya. ${ }^{2}$ Health Sciences Unit, Faculty of Social Sciences, University of Tampere, Tampere, Finland. ${ }^{3}$ Division of Disease Surveillance and Response, Ministry of Public Health and Sanitation, Nairobi, Kenya. ${ }^{4} \mathrm{WHO}$ Country Office for Kenya, Nairobi, Kenya. ${ }^{5} \mathrm{WHO}$ Regional Office for Africa (WHO/AFRO), Brazzaville, Congo. ${ }^{6}$ Division of Viral Diseases, US Centers for Disease Control and Prevention, Atlanta, GA, USA. ${ }^{7}$ Global Health Institute, Emory University, Atlanta, GA, USA. 
Received: 29 March 2018 Accepted: 12 December 2018

Published online: 11 January 2019

\section{References}

1. Parashar, U.D., et al., Health impact of rotavirus vaccination in developing countries: Progress and way forward. Clin Infect Dis, 2016. 62 Suppl 2: p. S91-5.

2. Kotloff $\mathrm{KL}$, et al. Burden and aetiology of diarrhoeal disease in infants and young children in developing countries (the global enteric multicenter study, GEMS): a prospective, case-control study. Lancet. 2013;382(9888):209-22.

3. Tate JE, et al. Global, regional, and National Estimates of rotavirus mortality in children < 5 years of age, 2000-2013. Clin Infect Dis. 2016;62(Suppl 2):S96-S105.

4. Tate, J.E., et al., Rotavirus disease burden and impact and cost-effectiveness of a rotavirus vaccination program in Kenya. J Infect Dis, 2009. 200 Suppl 1: p. S76-84.

5. WHO, Rotavirus vaccines WHO position paper: January 2013 recommendations. Vaccine, 2013. 31(52): p. 6170-1.

6. Gastanaduy PA, et al. Effect of rotavirus vaccine on diarrhea mortality in different socioeconomic regions of Mexico. Pediatrics. 2013;131(4):e1115-20.

7. Constenla DO, et al. Economic impact of a rotavirus vaccine in Brazil. $J$ Health Popul Nutr. 2008;26(4):388-96.

8. Lamberti LM, et al. A systematic review of the effect of rotavirus vaccination on diarrhea outcomes among children younger than 5 years. Pediatr Infect Dis J. 2016;35(9):992-8

9. Armah $\mathrm{GE}$, et al. Efficacy of pentavalent rotavirus vaccine against severe rotavirus gastroenteritis in infants in developing countries in sub-Saharan Africa: a randomised, double-blind, placebo-controlled trial. Lancet. 2010; 376(9741):606-14.

10. Bar-Zeev $\mathrm{N}$, et al. Effectiveness of a monovalent rotavirus vaccine in infants in Malawi after programmatic roll-out: an observational and case-control study. Lancet Infect Dis. 2015;15(4):422-8.

11. Gurgel RG, et al. Incidence of rotavirus and all-cause diarrhea in Northeast Brazil following the introduction of a national vaccination program. Gastroenterology. 2009;137(6):1970-5.

12. Armah, G., et al., Impact and effectiveness of monovalent rotavirus vaccine against severe rotavirus diarrhea in Ghana. Clin Infect Dis, 2016. 62 Suppl 2: p. S200-7.

13. Ngabo F, et al. Can routinely collected national data on childhood morbidity and mortality from diarrhea be used to monitor health impact of rotavirus vaccination in Africa? Examination of pre-vaccine baseline data from Rwanda. Pediatr Infect Dis J. 2014;33(Suppl 1):S89-93.

14. Rheingans $\mathrm{RD}$, et al. Economic costs of rotavirus gastroenteritis and costeffectiveness of vaccination in developing countries. J Infect Dis. 2009; 200(Suppl 1):S16-27.

15. Khagayi $\mathrm{S}$, et al. High burden of rotavirus gastroenteritis in young children in rural western Kenya, 2010-2011. Pediatr Infect Dis J. 2014;33(Suppl 1):S34-40.

16. Odhiambo FO, et al. Profile: the KEMRI/CDC health and demographic surveillance system--Western Kenya. Int J Epidemiol. 2012;41(4):977-87.

17. Mwenda JM, et al. Preparing for the scale-up of rotavirus vaccine introduction in Africa: establishing surveillance platforms to monitor disease burden and vaccine impact. Pediatr Infect Dis J. 2014;33(Suppl 1):S1-5.

18. Ruuska T, Vesikari T. Rotavirus disease in Finnish children: use of numerical scores for clinical severity of diarrhoeal episodes. Scand J Infect Dis. 1990; 22(3):259-67.

19. Omore R, et al. Health care-seeking behavior during childhood diarrheal illness: results of health care utilization and attitudes surveys of caretakers in western Kenya, 2007-2010. Am J Trop Med Hyg. 2013;89(1 Suppl):29-40.

20. Abramson JH. WINPEPI updated: computer programs for epidemiologists, and their teaching potential. Epidemiol Perspect Innov. 2011;8(1):1.

21. Long, J.X.a.J.S., Confidence Intervals for Predicted Outcomes in Regression Models for Categorical Outcomes, 2005 (Accessed online on November 16 2016). 2005

22. WHO, World Health Organization. Generic protocol for monitoring impact of rotavirus vaccination on gastroenteritis disease burden and viral strains: Geneva: World Health Organization, 2008; ( Accessed online on November 13, 2016). 2008.

23. Amek NO, et al. Childhood cause-specific mortality in rural Western Kenya: application of the InterVA-4 model. Glob Health Action. 2014;7:25581.

24. Byass $P$, et al. Strengthening standardised interpretation of verbal autopsy data: the new InterVA-4 tool. Glob Health Action. 2012;5:1-8.
25. Breiman RF, et al. Use of population-based surveillance to determine the incidence of rotavirus gastroenteritis in an urban slum and a rural setting in Kenya. Pediatr Infect Dis J. 2014;33(Suppl 1):S54-61.

26. Mustafa A, et al. Baseline burden of rotavirus disease in Sudan to monitor the impact of vaccination. Pediatr Infect Dis J. 2014;33(Suppl 1):S23-7.

27. Nokes DJ, et al. Rotavirus genetic diversity, disease association, and temporal change in hospitalized rural Kenyan children. J Infect Dis. 2010; 202(Suppl):S180-6.

28. Plenge-Bonig A, et al. Breastfeeding protects against acute gastroenteritis due to rotavirus in infants. Eur J Pediatr. 2010;169(12):1471-6.

29. Temu A, et al. Prevalence and factors associated with group a rotavirus infection among children with acute diarrhea in Mwanza, Tanzania. J Infect Dev Ctries. 2012;6(6):508-15.

30. Abebe A, et al. Hospital-based surveillance for rotavirus gastroenteritis in children younger than 5 years of age in Ethiopia: 2007-2012. Pediatr Infect Dis J. 2014;33(Suppl 1):S28-33.

31. Ministry Of Public Health and Sanitation, KENYA DEMOGRAPHIC AND HEALTH SURVEY (KDHS), 2014. Nairobi, Kenya.

32. Kovacs SD, et al. Deconstructing the differences: a comparison of GBD 2010 and CHERG's approach to estimating the mortality burden of diarrhea, pneumonia, and their etiologies. BMC Infect Dis. 2015;15:16.

33. Omore R, et al. Epidemiology, seasonality and factors associated with rotavirus infection among children with moderate-to-severe diarrhea in rural Western Kenya, 2008-2012: the global enteric multicenter study (GEMS). PLoS One. 2016;11(8):e0160060.

34. Bonkoungou IJ, et al. Epidemiology of rotavirus infection among young children with acute diarrhoea in Burkina Faso. BMC Pediatr. 2010;10:94.

35. WHO, THE TREATMENT OF DIARRHOEA. A manual for physicians and other senior health workers, 4th revision. World Health Organization (WHO) press, 2005.

36. WHO, WHO: External review of burden of disease attributable to rotavirus. 2005.

37. Ouedraogo N, et al. Temporal distribution of gastroenteritis viruses in Ouagadougou, Burkina Faso: seasonality of rotavirus. BMC Public Health. 2017;17(1):274.

38. Tate JE, et al. Sustained decline in rotavirus detections in the United States following the introduction of rotavirus vaccine in 2006. Pediatr Infect Dis J. 2011;30(1 Suppl):S30-4.

39. Colston JM, et al. Seasonality and within-subject clustering of rotavirus infections in an eight-site birth cohort study. Epidemiol Infect. 2018;146(6):688-97.

40. Patel MM, et al. Global seasonality of rotavirus disease. Pediatr Infect Dis J. 2013;32(4):e134-47.

41. ljaz MK, et al. Effect of relative humidity, atmospheric temperature, and suspending medium on the airborne survival of human rotavirus. Can J Microbiol. 1985;31(8):681-5.

42. Cook SM, et al. Global seasonality of rotavirus infections. Bull World Health Organ. 1990;68(2):171-7.

43. Sattar SA, et al. Effect of relative humidity on the airborne survival of rotavirus SA11. Appl Environ Microbiol. 1984;47(4):879-81.

44. Ruiz-Palacios GM, et al. Safety and efficacy of an attenuated vaccine against severe rotavirus gastroenteritis. N Engl J Med. 2006;354(1):11-22.

45. Groome MJ, et al. Effectiveness of monovalent human rotavirus vaccine against admission to hospital for acute rotavirus diarrhoea in south African children: a case-control study. Lancet Infect Dis. 2014;14(11):1096-104.

46. Tsolenyanu, E., et al., Early evidence of impact of monovalent rotavirus vaccine in Togo. Clin Infect Dis, 2016. 62 Suppl 2: p. S196-S199.

Ready to submit your research? Choose BMC and benefit from:

- fast, convenient online submission

- thorough peer review by experienced researchers in your field

- rapid publication on acceptance

- support for research data, including large and complex data types

- gold Open Access which fosters wider collaboration and increased citations

- maximum visibility for your research: over $100 \mathrm{M}$ website views per year

At BMC, research is always in progress.

Learn more biomedcentral.com/submissions 